\title{
A Single Coronary Artery with an Absent Right Coronary and a Superdominant Left Circumflex Giving off a Branch Supplying the Right Coronary Artery Territory
}

\author{
Imran Iftikhar, Adeel Ur Rehman and Hamid Sharif Khan \\ Rawalpinidi Institute of Cardiology, Rawalpindi, Pakistan
}

\begin{abstract}
An absent right coronary artery (RCA) with single left coronary artery (LCA) originating from left aortic sinus with a superdominant left circumflex (LCX) and giving off an RCA branch is one of the rarest coronary artery anomalies. It occurs with an incidence of less than $0.1 \%$. Usually, patients are asymptomatic and abnormality is found incidentally on cardiac catheterisation or CT angiography. We present a case report of an unusual coronary anomaly in a patient who presented with anterior myocardial infarction. Patient was subjected to coronary angiography, which revealed absent RCA originating from distal LCX artery, supplying the base of heart and RCA territory. He underwent primary percutaneous intervention $(\mathrm{PCl})$ with stenting to the left anterior descending (LAD) artery. This type of anomaly, in which single LCA from which dominant LCX continues as RCA, is important to diagnose and manage if diseased, as stenosis of the dominant LCX artery in such cases can jeopardise a large portion of myocardium, which can lead to increased morbidity and mortality, if left untreated.
\end{abstract}

Key Words: Myocardial infarction, Coronary anomaly, Coronary angiography.

How to cite this article: Iftikhar I, Rehman AU, Khan HS. A single coronary artery with an absent right coronary and a superdominant left circumflex giving off a branch supplying the right coronary artery territory. J Coll Physicians Surg Pak 2019; 29 (Supplement 2):S80-S82.

\section{INTRODUCTION}

Single left coronary artery (LCA) arising from the aorta as a single ostium, supplying blood to all the myocardium, is a rare coronary anomaly. ${ }^{1}$ Its prevalence in literature ranges between $0.024 \%^{2}$ to $0.066 \%^{3}$ in those who undergo cardiac catheterization. There are different classification systems of single coronary artery based on angiographic data and autopsy findings. ${ }^{4} \mathrm{~A}$ very informative and useful classification was proposed by Lipton et al. ${ }^{1}$ which describes the site of origin and branches of single coronary artery based on the site of origin from aortic sinuses; right and left type are described as primary divisions. This type of anomaly in which single LCA from which dominant left circumflex (LCX) artery continues as right coronary artery (RCA) is important to diagnose and manage if diseased; as stenosis of the dominant LCX artery in such cases can jeopardize a large portion of myocardium which can lead to increased morbidity and mortality, if left untreated.

Single LCA with congenital absence of RCA is extremely rare and very few case reports have been published in the literature.

Correspondence to: Dr. Imran Iftikhar, Rawalpinidi Institute

of Cardiology, Rawalpindi, Pakistan

E-mail: imraniftikhar1519@yahoo.com

Received: February 04, 2019; Revised: July 21, 2019;

Accepted: August 06, 2019
Herein, we present a case of a 45-year male who had single LCA, that was incidentally found on coronary angiography; done for the investigation of anterior myocardial infarction.

\section{CASE REPORT}

A 45-year male, hypertensive and smoker, presented with complaints of chest pain and worsening shortness of breath over the last three days. Electrocardiogram (ECG) showed $Q$ waves in the anterior leads with ST segment elevation. He was diagnosed as anterior wall ST elevation myocardial infarction (anterior STEMI), late for lytic therapy. His coronary angiogram was done which revealed critical stenosis in mid-course of left anterior descending (LAD) artery and LCX artery continued as RCA (Figures 1 and 2). We searched for the RCA by taking an aortogram in the aortic root but could not see any vessel originating from the right sinus, thus concluding that it was congenitally absent (Figure 3). An aberrant branch, continued from the dominant LCX artery crossing the crux and continuing its course in right atrioventricular groove supplying the RCA territory, was evident. This anomalous branch from LCX artery terminated near the right sinus of Valsalva.

Left ventriculogram showed severe left ventricular systolic dysfunction. The patient was advised myocardial perfusion imaging (MPI) scan to assess the viability of the myocardium. 


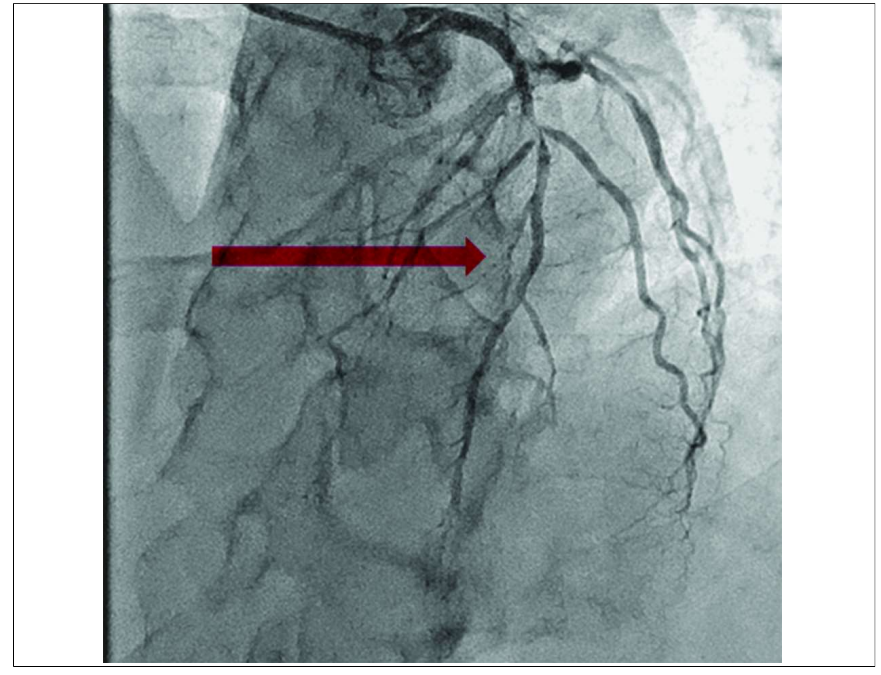

Figure 1: Coronary angiogram showing left anterior descending artery.

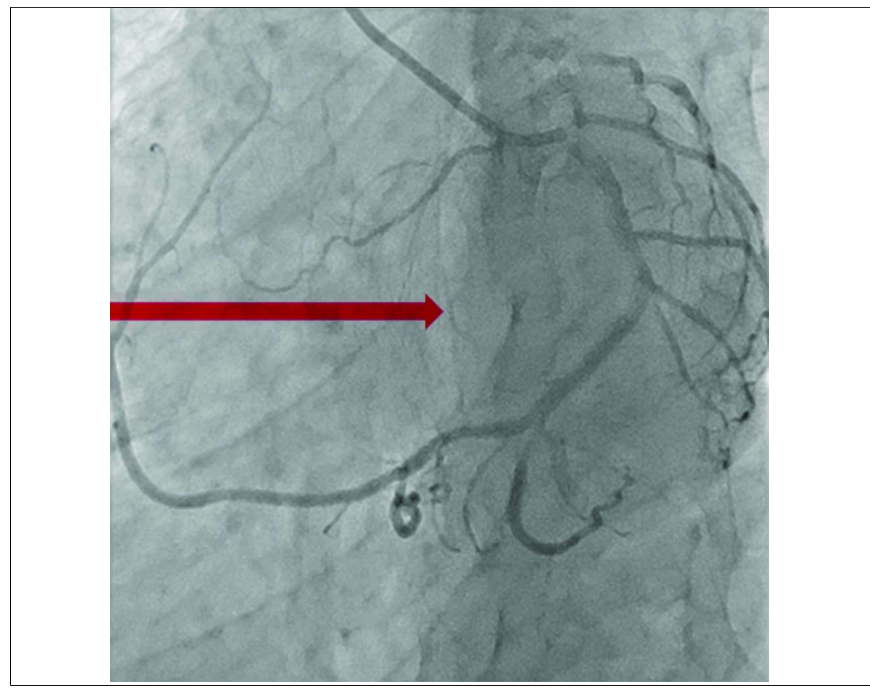

Figure 2: Coronary angiogram showing dominant left circumflex artery continuing as right coronary artery.

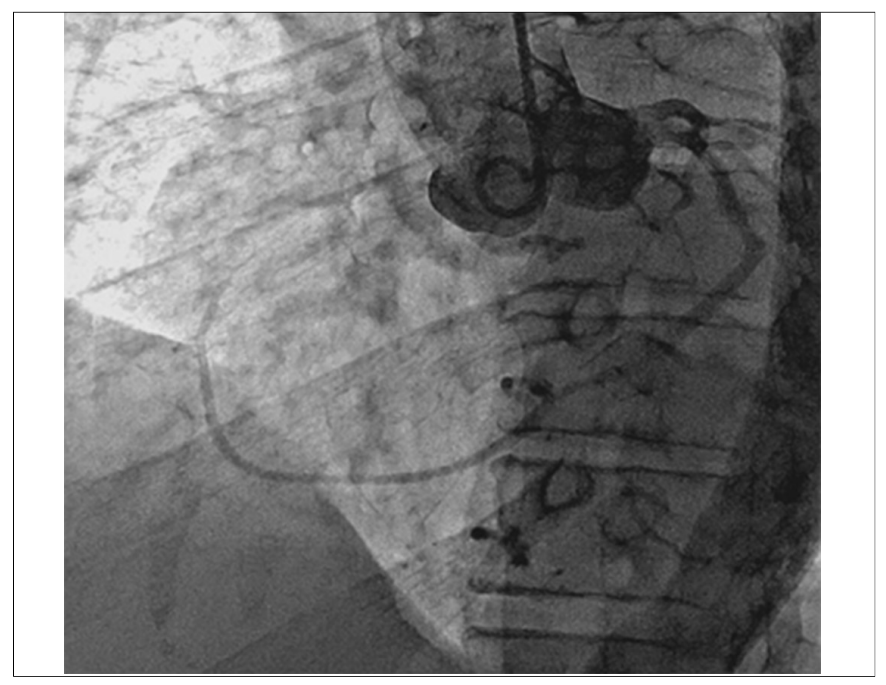

Figure 3: Aortogram showing absence of origin of the right coronary artery.

\section{DISCUSSION}

According to Lipton classification, there are 9 types of single coronary arteries based on the site of origin and their anatomical course. ${ }^{1}$ RCA originating from distal LCX artery is a very rare coronary anomaly and only 24 cases have been reported in literature to date, comprising 15 male and 9 female patients. 5 According to the Lipton classification, all these patients had L1 type coronary anomaly where the right coronary ostium was absent and LCA bifurcated into LAD and LCX, and distal LCX artery continued as right coronary beyond the crux into atrioventricular groove till the base of heart.6 The coronary anomaly under discussion, i.e., L1 type, usually runs a benign course as observed in the cases reported in literature, although the single coronary artery can be associated with other congenital anomalies and with the development of cardiomyopathy, heart failure and arrhythmias. 7,8

The outcomes of such a coronary anomaly are quite variable in literature. Most of the reported cases have had negative ischemic workup with the anomalous RCA running a benign course. Choi et al. 9 reported a case, which presented with chest discomfort. Coronary angiogram showed single LCA and patient's chest discomfort was attributed to the sluggish flow in RCA. Patient was treated with calcium channel blockers and nitrates. Ma et al.10 described a patient whose stenting was done in distal LCX as he presented with inferior wall myocardial infarction. Ghaffari et al. ${ }^{11}$ reported a patient who had massive pulmonary embolism and had single LCA on coronary angiography. The patient had prolonged hemodynamic instability which was attributed to prolonged and disproportionate right ventricular dysfunction due to its compromised perfusion in the setting of acute pulmonary hypertension and absence of proximal RCA. ${ }^{11}$ Other cardiac conditions have been associated with this rare coronary anomaly such as atrial fibrillation (AF) and mitral regurgitation (MR), which were described in one patient in the literature. However, coronary artery disease in cases where only one coronary artery is present becomes significant as these patients are dependent on only single artery.

Our patient did not have evidence of ischemia in RCA territory. In fact, the patient presented with anterior STEMI and coronary angiogram showing critical disease in LAD and the distal LCX, which continued as RCA, was free from disease. The patient had primary $\mathrm{PCl}$ (stenting) to the LAD.

In conclusion, this case highlights the need for keeping in mind that the various anomalies of coronary arteries during the investigation of myocardial infarction, as these may affect the outcome of ischemic insult.

\section{PATIENT'S CONSENT:}

Informed consent was taken from the patient to publish his case as a case report. 


\section{CONFLICT OF INTEREST:}

Authors declared no conflict of interest.

AUTHORS' CONTRIBUTION:

II: Performed the case, took images, wrote the case report. AUR: Worked on the references.

HSK: Contributed in the Introduction and Discussion.

\section{REFERENCES}

1. Lipton MJ, Barry WH, Obrez I, Silverman JF, Wexler L. Isolated single coronary artery: Diagnosis, angiographic classification, and clinical significance. Radiology 1979; 130:39-47.

2. Desmet W, Vanhaecke J, Vrolix M, Van de Werf F, Piessens J, Willems $\mathrm{J}$, et al. Isolated single coronary artery: A review of 50,000 consecutive coronary angiographies. Eur Heart J 1992; 13:1637-40.

3. Yamanaka O, Hobbs RE. Coronary artery anomalies in 126,595 patients undergoing coronary arteriography. Catheter Cardiovasc Diagn 1990; 21:28-40.

4. Said SA, De Vooqt WG, Bulut S, Han J, Polak P, Nijhuis RL, et al. Coronary artery disease in congenital single coronary artery in adults: A Dutch case series. World J Cardiol 2014; 6:196-204.

5. Tavernarakis A, Voudris V, Ifantis G, Tsaganos N. Anomalous origin of the right coronary artery arising from the circumflex artery. Clin Cardiol 1986; 9:230-2.

6. Asha M, Sriram R, Mukundan S, Abraham K. Single coronary artery from the left sinus with atherosclerosis. Asian Cardiovasc Thorac Ann 2003; 11:163.

7. Datta S, Moussa T, Hussain F. Anomalous right coronary artery originating from the distal left circumflex artery: A novel coronary artery anomaly viewed by computed tomography and invasive angiography. Can J Cardiol 2010; 26:213.

8. Yoshimoto S, Hirooka K, Irino H, Abe H, Yasuoka Y, Yamamoto H. et al. Anomalous right coronary artery originating from distal left circumflex artery in a patient with an unusual type of isolated single coronary artery. Jpn Heart J 2004; 45:337-42.

9. Choi HY, Kim JW, Moon JM, Kim YJ, Choi CU, Lim HE. et al. Unusual dominant course of left circumflex coronary artery to right coronary artery territory with absent right coronary artery. J Cardiol 2010; 55:117-9.

10. Ma SH, Kim DH, Hur JY, Kim KS, Byun SJ, Park KH. et al. Right ventricular myocardial infarction due to right coronary artery total occlusion originating from the distal left circumflex artery. Korean Circ J 2012; 42:565-7.

11. Ghaffari S, Pourafkari L. Pulmonary embolism in a patient with a rare coronary anomaly - a clue to the importance of proximal right coronary artery branches. Kardiol Pol 2010; 68:844-6. 